\title{
Disconnected Forbidden Subgraphs, Toughness and Hamilton Cycles
}

\author{
Zh. G. Nikoghosyan \\ Institute for Informatics and Automation Problems, National Academy of Sciences of the Republic of Armenia, \\ 1 P. Sevak Street, 0014 Yerevan, Armenia \\ Correspondence should be addressed to Zh. G. Nikoghosyan; zhora@ipia.sci.am
}

Received 2 October 2012; Accepted 18 November 2012

Academic Editors: M. Alekseyev, M. Eliasi, N. A. Gordon, A. V. Kelarev, C.-K. Lin, V. Y. Protasov, and Y. Zhang

Copyright (c) 2013 Zh. G. Nikoghosyan. This is an open access article distributed under the Creative Commons Attribution License, which permits unrestricted use, distribution, and reproduction in any medium, provided the original work is properly cited.

In 1974, Goodman and Hedetniemi proved that every 2 -connected $\left(K_{1,3}, K_{1,3}+e\right)$-free graph is hamiltonian. This result gave rise many other conditions for Hamilton cycles concerning various pairs and triples of forbidden connected subgraphs under additional connectivity conditions. In this paper we investigate analogous problems when forbidden subgraphs are disconnected which affects more global structures in graphs such as tough structures instead of traditional connectivity structures. In 1997, it was proved that a single forbidden connected subgraph $R$ in 2-connected graphs can create only a trivial class of hamiltonian graphs (complete graphs) with $R=P_{3}$. In this paper we prove that a single forbidden subgraph $R$ can create a non trivial class of hamiltonian graphs if $R$ is disconnected: $(* 1)$ every $\left(K_{1} \cup P_{2}\right)$-free graph either is hamiltonian or belongs to a well defined class of non hamiltonian graphs; $(* 2)$ every 1-tough $\left(K_{1} \cup P_{3}\right)$-free graph is hamiltonian. We conjecture that every 1-tough $\left(K_{1} \cup P_{4}\right)$-free graph is hamiltonian and every 1-tough $P_{4}$-free graph is hamiltonian.

\section{Introduction}

Only finpite undiprected graphs without loops or multiple edges are considered. Let $G$ be a graph with vertex set $V(G)$ and edge set $E(G)$. We write $G[S]$ for the subgraph of $G$ induced by $S$. The neighborhood of a vertex $x \in V(G)$ will be denoted by $N(x)$. We use $n$ to denote the number of vertices (order) of $G$. The independence number of $G$, denoted by $\alpha$, is the maximum size of an mutually nonadjacent vertices in $G$. If $H_{1}, \ldots, H_{t}(t \geq 1)$ are graphs then a graph $G$ is said to be $\left(H_{1}, \ldots, H_{t}\right)$-free if $G$ contains no copy of any of the graphs $H_{1}, \ldots, H_{t}$ as an induced subgraph; the graphs $H_{1}, \ldots, H_{t}$ will be also referred to in this context as forbidden subgraphs. We denote by $P_{i}$ and $C_{i}$ the path and the cycle on $i$ vertices. Further, $K_{n}$ denotes the complete graph of order $n$, and $K_{a, b}$ denotes the complete bipartite graph with partite sets of cardinalities $a$ and $b$. Specifically, the graph $K_{1,3}$ will be called a claw. The graph $K_{1,3}+e$ is obtained from $K_{1,3}$ by adding an edge. Let $N_{i, j, k}$ be the graph which is obtained by identifying each vertex of a triangle with an end vertex of one of three vertex-disjoint paths of lengths $i, j, k$. Let $s(G)$ denote the number of components of a graph $G$. A graph $G$ is $t$-tough if $|S| \geq t s(G \backslash S)$ for every subset $S$ of the vertex set $V(G)$ with $s(G \backslash S)>1$. The toughness of $G$, denoted $\tau(G)$, is the maximum value of $t$ for which $G$ is $t$-tough (taking $\tau\left(K_{n}\right)=$ $\infty$ for all $n \geq 1$ ). A good reference for any undefined terms is [1].

The first sufficient condition for hamiltonicity of a graph in terms of forbidden subgraphs is by Goodman and Hedetniemi [2].

Theorem A (see [2]). Every 2-connected $\left(K_{1,3}, K_{1,3}+e\right)$-free graph is hamiltonian.

This result gave rise to many other hamiltonicity conditions for various pairs and triples of forbidden connected subgraphs under additional connectivity conditions.

In 1997, Faudree and Gould [3] proved that a single forbidden connected subgraph $R$ in 2-connected graphs can create only a trivial class (complete graphs) of hamiltonian graphs with $R=P_{3}$. 
Theorem B (see [3]). Let $R$ be a connected graph and let $G$ be a 2-connected graph. Then $G$ is $R$-free implies $G$ is hamiltonian if and only if $R=P_{3}$.

The following theorem presents the forbidden pairs version of Theorem $B$.

Theorem C (see [3]). Let $R$ and $S$ be connected graphs $\left(R, S \neq P_{3}\right)$ and $G$ be a 2 -connected graph of order $n \geq 10$. Then $G$ is $(R, S)$-free implies $G$ is Hamiltonian if and only if $R=K_{1,3}$ and $S$ is one of the graphs:

$$
P_{4}, P_{5}, P_{6}, N_{0,0,0}, N_{0,0,1}, N_{0,0,2}, N_{0,1,1}, N_{0,0,3}, N_{0,1,2}, N_{1,1,1} \text {. }
$$

The forbidden triple version of Theorems B and C still is not known.

In this paper we investigate analogous problems when forbidden subgraphs are disconnected which affects more global structures in graphs such as tough structures instead of traditional connectivity structures.

First of all, we prove that a single forbidden subgraph $R$ can create a non trivial class of hamiltonian graphs if $R$ is disconnected. As starting point, we consider $K_{1} \cup K_{1}$ and $K_{1} \cup K_{1} \cup K_{1}$ as disconnected sugraphs consisting of two and three isolated vertices, respectively. In other words, $K_{1} \cup K_{1}$ is a compliment of $K_{2}$ and $K_{1} \cup K_{1} \cup K_{1}$ is a complement of $K_{3}$ (a triangle). The first proposition follows immediately.

Proposition 1. Every $\left(K_{1} \cup K_{1}\right)$-free graph is complete and therefore is hamiltonian.

The next proposition shows that $\left(K_{1} \cup K_{1} \cup K_{1}\right)$-free graphs are hamiltonian if and only if they are 2 -connected.

Proposition 2. Every 2-connected $\left(K_{1} \cup K_{1} \cup K_{1}\right)$-free graph is hamiltonian.

Observe that $K_{1} \cup P_{2}$ is the minimal forbidden disconnected subgraph containing at least one edge. To describe the hamiltonian graphs with forbidden subgraph $\left(K_{1} \cup P_{2}\right)$, we need the following recursive definition.

Definition 3. We say that $G \in \aleph$ if and only if either $V(G)$ is independent set of vertices or $G$ is complete graph or there is a bipartition $V(G)=V_{1} \cup V_{2}$ such that

(1) $N(v)=V_{2}$ for each $v \in V_{1}$,

(2) $G\left[V_{2}\right] \in \aleph$.

By the Definition, if $G$ is a complete bipartite graph then $G \epsilon$ $\aleph$.

Theorem 4. If $G$ is a $\left(K_{1} \cup P_{2}\right)$-free graph then either $G$ is Hamiltonian or $G \in \aleph$ with $\alpha(G)>n / 2$.

If $G$ is a 1-tough graph then by the definition, $\alpha(G) \leq n / 2$. Hence, the following holds from Theorem 4 immediately.

Corollary 5. Every 1-tough $\left(K_{1} \cup P_{2}\right)$-free graph is hamiltonian.

This result can be slightly improved.
Theorem 6. Every 1-tough $\left(K_{1} \cup P_{3}\right)$-free graph is hamiltonian.

Theorem 6 is sharp in some respects. Indeed, $K_{2,3}$ is a nonhamiltonian $\left(K_{1} \cup P_{3}\right)$-free (even $\left(K_{1} \cup P_{2}\right)$-free) graph with $\tau\left(K_{2,3}\right)=2 / 3$ and $\kappa\left(K_{2,3}\right)=2$, implying that the condition " $G$ is 1-tough" in Theorem 6 cannot be removed or replaced by " $G$ is 2-connected." Now form a graph, denoted by $H_{1}$, by adding a new vertex $x_{7}$ to $C_{6}=x_{1} x_{2} x_{3} x_{4} x_{5} x_{6} x_{1}$ and new edges $x_{7} x_{1}, x_{7} x_{4}, x_{2} x_{6}, x_{2} x_{4}, x_{4} x_{6}$. Since $H_{1}$ is a nonhamiltonian $\left(K_{2} \cup P_{3}\right)$-free graph with $\tau\left(H_{1}\right)=1$, we can claim that the condition " $G$ is $\left(K_{1} \cup P_{3}\right)$-free" in Theorem 6 cannot be relaxed to " $G$ is $\left(K_{2} \cup P_{3}\right)$-free." Finally, $H_{1}$ is a $\left(K_{1} \cup K_{1,3}\right)$-free graph and hence the condition " $G$ is $\left(K_{1} \cup P_{3}\right)$ free" in Theorem 6 cannot be relaxed to " $G$ is $\left(K_{1} \cup K_{1,3}\right)$ free." So, Theorem 6 is best possible in many respects. The condition " $G$ is $\left(K_{1} \cup P_{3}\right)$-free" in Theorem 6 perhaps can be relaxed to " $G$ is $\left(K_{1} \cup P_{4}\right)$-free."

Conjecture 7. Every 1-tough $\left(K_{1} \cup P_{4}\right)$-free graph is hamiltonian.

Conjecture 7 (if true) is best possible in all respects. Indeed, the above constructed graph $H_{1}$ shows that the condition " $G$ is $\left(K_{1} \cup P_{4}\right)$-free" in Conjecture 7 cannot be replaced by " $G$ is $\left(K_{1} \cup P_{5}\right)$-free."

Moreover, the following seems reasonable as well.

Conjecture 8. Every 1-tough $P_{4}$-free graph is hamiltonian.

For more than one tough graphs, we conjecture the following.

Conjecture 9. Every $\left(K_{1} \cup P_{5}\right)$-free graph with $\tau>1$ is hamiltonian.

The Petersen graph shows that the condition " $G$ is $\left(K_{1} \cup\right.$ $\left.P_{5}\right)$-free" in Conjecture 9 cannot be replaced by " $G$ is $\left(K_{1} \cup P_{6}\right)$ free".

The next conjecture concerns $\left(K_{2} \cup K_{2}\right)$-free graphs.

Conjecture 10. Every $\left(K_{2} \cup K_{2}\right)$-free graph with $\tau>1$ is hamiltonian.

The graph $H_{1}$ (see the graph examples concerning the sharpness of Theorem 6) shows that the condition $\tau>1$ in Conjecture 10 can not be replaced by $\tau=1$. Further, the Petersen graph shows that the condition " $G$ is $\left(K_{2} \cup K_{2}\right)$-free" in Conjecture 10 can not be replaced by " $G$ is $\left(K_{2} \cup K_{3}\right)$-free".

The next conjecture is based on a forbidden subgraph $K_{1} \mathrm{U}$ $K_{1,3}$ containing the claw $K_{1,3}$ as a substructure.

Conjecture 11. Every $\left(K_{1} \cup K_{1,3}\right)$-free graph with $\tau>4 / 3$ is hamiltonian.

The Petersen graph shows that the condition $\tau>4 / 3$ in Conjecture 11 can not be replaced by $\tau=4 / 3$.

\section{Additional Notation and Preliminaries}

For $S$ a subset of $V(G)$, we denote by $G \backslash S$ the subgraph of $G$ induced by $V(G) \backslash S$. For a subgraph $H$ of $G$ we use $G \backslash H$ 
short for $G \backslash V(H)$. Furthermore, for a subgraph $H$ of $G$ and $x \in V(G)$, we define $N_{H}(x)=N(x) \cap V(H)$.

A simple cycle (or just a cycle) $C$ of length $t \geq 2$ is a sequence $v_{1} v_{2} \cdots v_{t} v_{1}$ of distinct vertices $v_{1}, \ldots, v_{t}$ with $v_{i} v_{i+1} \in E(G)$ for each $i \in\{1, \ldots, t\}$, where $v_{t+1}=v_{1}$. When $t=2$, the cycle $C=v_{1} v_{2} v_{1}$ on two vertices $v_{1}, v_{2}$ coincides with the edge $v_{1} v_{2}$. A graph $G$ is hamiltonian if $G$ contains a Hamilton cycle, that is, a cycle of length $n$. A cycle $C$ in $G$ is dominating if $G \backslash C$ is edgeless.

Paths and cycles in a graph $G$ are considered as subgraphs of $G$. If $Q$ is a path or a cycle, then the length of $Q$, denoted by $|Q|$, is $|E(Q)|$. We write $Q$ with a given orientation by $\vec{Q}$. For $x, y \in V(Q)$, we denote by $x \vec{Q} y$ the subpath of $Q$ in the chosen direction from $x$ to $y$. The expression $Q=x \vec{Q} y$ means that $x, y$ are the endvertices of $Q$. For $x \in V(C)$, we denote the $h$ th successor and the $h$ th predecessor of $x$ on $\vec{C}$ by $x^{+h}$ and $x^{-h}$, respectively. We abbreviate $x^{+1}$ by $x^{+}$. For each $X \subset V(C)$, we define $X^{+h}=\left\{x^{+h} \mid x \in X\right\}$.

To prove Proposition 2 and Theorems 4, 6, we need the following useful lemma.

Lemma 12. Let $G$ be a 2-connected graph, $\vec{C}$ a longest cycle in $G$ with a given orientation and $x \in V(G \backslash C)$. Let $\vec{P}_{1}, \vec{P}_{2}$ be two internally disjoint paths with given orientations starting at $x$ and terminating on $C$ at different vertices $u, v$. Then $u^{+} v^{+} \notin$ $E(G)$.

\section{Proofs}

Proof of Lemma 12. Assume the contrary, that is $u^{+} v^{+} \in$ $E(G)$. Then

$$
u \overleftarrow{P_{1}} x \vec{P}_{2} v \overleftarrow{C} u^{+} v^{+} \vec{C} u
$$

is longer than $C$, contradicting the fact that $C$ is a longest cycle in $G$. Hence, $u^{+} v^{+} \notin E(G)$. Lemma 12 is proved.

Proof of Proposition 2. Since $G$ is 2-connected graph, it contains a cycle. Let $\vec{C}$ be a longest cycle in $G$ with a given orientation. If $V(G \backslash C)=\emptyset$ then $C$ is a Hamilton cycle and we are done. Let $V(G \backslash C) \neq \emptyset$ and let $x \in V(G \backslash C)$. Since $G$ is 2-connected, there are two internally disjoint paths $P_{1}, P_{2}$ with given orientations starting at $x$ and terminating on $C$ at different vertices $u, v$. If $x u^{+} \in E(G)$ then $u \overleftarrow{P_{1}} x u^{+} \vec{C} u$ is longer than $C$, contradicting the fact that $C$ is a longest cycle. Hence, $x u^{+} \notin E(G)$. Analogously, $x v^{+} \notin E(G)$. Furthermore, by Lemma $12, u^{+} v^{+} \notin E(G)$, implying that $\left\{x, u^{+}, v^{+}\right\}$is an independent set of vertices which contradicts the fact that $G$ is $\left(K_{1} \cup K_{1} \cup K_{1}\right)$-free graph. This final contradiction proves that $G$ is hamiltonian. Proposition 2 is proved.

Proof of Theorem 4. Let $G$ be a $\left(K_{1} \cup P_{2}\right)$-free graph. If $V(G)$ is independent set of vertices, then by the definition, $G \in \aleph$ with $\alpha(G)=n>n / 2$ and we are done. Let $G$ contain at least one edge. Next, if $G$ is disconnected, then clearly $G$ contains $K_{1} \cup P_{2}$ as an induced subgraph, contradicting the hypothesis. Let $G$ be connected. Further, if $G$ is a tree, then clearly $G$ either is a star (i.e., $G$ is a complete bipartite graph and hence $G \in \aleph$ with $\alpha(G)=n-1>n$ ) or contains $K_{1} \cup P_{2}$ as an induced subgraph, again contradicting the hypothesis. Now assume that $G$ is not a tree and let $C=v_{1} v_{2} \ldots v_{t} v_{1}$ be a longest cycle in $G$ with a given orientation. If $V(G \backslash C)=\emptyset$, then $C$ is a Hamilton cycle and we are done. Let $V(G \backslash C) \neq \emptyset$. Since $G$ is connected, we have $x y \in E(G)$ for some $x \in V(G \backslash C)$ and $y \in$ $V(C)$. Assume without loss of generality that $y=v_{1}$. Since $C$ is a longest cycle, we have $x v_{2} \notin E(G)$. If $x v_{3} \notin E(G)$, then $x$ and $v_{2} v_{3}$ form an induced subgraph $K_{1} \cup P_{2}$, contradicting the hypothesis. Hence $x v_{3} \in E(G)$. By a similar argument, $x v_{i} \in$ $E(G)$ for each $i=1,3,5, \ldots, t-1$ and $x v_{i} \notin E(G)$ for each $i=2,4,6, \ldots, t$ where $t$ is even. Further, since $C$ is a longest cycle, by Lemma $12,\left\{x, v_{2}, v_{4}, \ldots, v_{t}\right\}$ is an independent set of vertices. Moreover, for each $u, v \in\left\{x, v_{2}, v_{4}, \ldots, v_{t}\right\}$, there is no path connecting $u$ and $v$ and passing through $V(G) \backslash$ $(V(C) \cup\{x\})$.

To prove that $N(x)=\left\{v_{1}, v_{3}, \ldots, v_{t-1}\right\}$, assume the contrary, that is $x z \in E(G)$ for some $z \in V(G) \backslash(V(C) \cup$ $\{x\})$. Recalling that $C$ is a longest cycle, we conclude that $z v_{2} \notin E(G)$ and $z v_{3} \notin E(G)$. Therefore, $z$ and $v_{2} v_{3}$ form a disconnected subgraph $K_{1} \cup P_{2}$ which contradicts the fact that $G$ is $\left(K_{1} \cup P_{2}\right)$-free. Hence $N(x) \subseteq V(C)$. But then it is not hard to see that $V(G \backslash C)$ is an independent set of vertices.

Now let us prove that $N(v)=\left\{v_{1}, v_{3}, \ldots, v_{t-1}\right\}$ for each $v \in$ $V(G \backslash C)$. Assume that $v \neq x$. Since $V(G \backslash C)$ is an independent set of vertices, we have $N(v) \subseteq V(C)$. if $v_{2} v \in E(G)$, then as above $v_{4} v \in E(G)$, implying that

$$
v_{1} x v_{3} v_{2} v v_{4} \vec{C} v_{1}
$$

is longer than $C$, a contradiction. Let $v_{2} v \notin E(G)$. But then (as above) $v_{1} v \in E(G)$, implying that $N(v)=\left\{v_{1}, v_{3}, \ldots, v_{t-1}\right\}$. Put

$$
V_{1}=V(G \backslash C) \cup\left\{v_{2}, v_{4}, \ldots, v_{t}\right\}, \quad V_{2}=\left\{v_{1}, v_{3}, \ldots, v_{t-1}\right\} .
$$

Clearly $V(G)=V_{1} \cup V_{2}$ Since $V_{1}$ is an independent set of vertices and $\left|V_{1}\right|>\left|V_{2}\right|$, we can state that $\alpha(G) \geq\left|V_{1}\right|>n / 2$ and thus $G$ is not hamiltonian. It remains to prove that $G \in \aleph$. If $V_{2}$ is independent or $G\left[V_{2}\right]$ is complete, then $G \in \aleph$ and we are done. Otherwise denote by $V_{3}$ a largest independent subset in $V_{2}$ and put $V_{4}=V_{2} \backslash V_{3}$. Clearly, $\left|V_{3}\right| \geq 2$ and $V_{4} \neq \emptyset$. Let $w_{2}$ be any vertex in $V_{4}$. Since $V_{3}$ is a largest independent set, there exists a vertex $w_{1} \in V_{3}$ such that $w_{1} w_{2} \in E(G)$. Let $w_{3} \in V_{3} \backslash\left\{w_{1}\right\}$. If $w_{2} w_{3} \notin E(G)$, then $w_{3}$ and $w_{1} w_{2}$ form an induced subgraph $K_{1} \cup P_{2}$, contradicting the hypothesis. Hence $w_{2} w_{3} \in E(G)$ implying that $w_{2} v \in E(G)$ for each $v \in$ $V_{3}$. This means also that each vertex in $V_{4}$ is adjacent to all vertices in $V_{3}$. Applying the same arguments to $V_{4}$ instead of $V_{2}$, we conclude that $G \in \aleph$.

Proof of Theorem 6. Let $G$ be a 1-tough $\left(K_{1} \cup P_{3}\right)$-free graph. In particular, $G$ is 2-connected graph and therefore contains a cycle. Let $C$ be a longest cycle in $G$ with a given orientation. If $V(G \backslash C)=\emptyset$ then $C$ is a Hamilton cycle and we are done. Let $V(G \backslash C) \neq \emptyset$ and let $H$ be a connected component of $G \backslash C$ of maximum order. Denote by $\xi_{1}, \ldots, \xi_{s}$ the elements of $N_{C}(H)$ occurring on $C$ in a consecutive order. Since $G$ is 1-tough, we have $s \geq 2$. Set

$$
I_{i}=\xi_{i} \vec{C} \xi_{i+1}, \quad I_{i}^{*}=\xi_{i}^{+} \vec{C} \xi_{i+1}^{-} \quad(i=1,2, \ldots, s),
$$


where $\xi_{s+1}=\xi_{1}$. Since $C$ is a longest cycle in $G$, we have $\left|V\left(I_{i}\right)\right| \geq 3$ and $\left|V\left(I^{*}{ }_{i}\right)\right| \geq 1$ for each $i \in\{1,2, \ldots, s\}$. The segments $I_{1}, I_{2}, \ldots, I_{s}$ are called elementary segments on $C$ induced by $N_{C}(H)$. We call a path $L=z \vec{L} w$ an intermediate path between two distinct elementary segments $I_{a}$ and $I_{b}$ if

$$
z \in V\left(I_{a}^{*}\right), w \in V\left(I_{b}^{*}\right), \quad V(L) \cap V(C \cup H)=\{z, w\} .
$$

Define $Y\left(I_{i_{1}}, I_{i_{2}}, \ldots, I_{i_{t}}\right)$ to be the set of all intermediate paths between elementary segments $I_{i_{1}}, I_{i_{2}}, \ldots, I_{i_{t}}$. If $\Upsilon\left(I_{1}, I_{2}, \ldots, I_{s}\right)=\emptyset$, then $G \backslash\left\{\xi_{1}, \ldots, \xi_{s}\right\}$ has at least $s+$ 1 connected components, contradicting the fact that $G$ is 1-tough. Otherwise $\Upsilon\left(I_{a}, I_{b}\right) \neq \emptyset$ for some distinct $a, b \in$ $\{1, \ldots, s\}$. Choose a path $L=x \vec{L} y$ in $\Upsilon\left(I_{a}, I_{b}\right)$ such that $x \in$ $V\left(I_{a}^{*}\right)$ and $y \in V\left(I_{b}^{*}\right)$. If $|V(L)| \geq 3$, then each vertex $v \in V(H)$ with the subpath of $L$ of length 2 forms an induced subgraph $K_{1} \cup P_{3}$, contradicting the hypothesis. Let $|V(L)|=2$, that is, $L=x y$ and hence $\Upsilon\left(I_{1}, I_{2}, \ldots, I_{s}\right) \subseteq E(G)$. Put

$$
Q=\xi_{a}^{+} \vec{C} x y \overleftarrow{C} \xi_{b}^{+}
$$

Assume without loss of generality that $L$ is chosen from $\Upsilon\left(I_{a}, I_{b}\right)$ such that $|V(Q)|$ is minimum. Since $C$ is a longest cycle in $G$, by Lemma $12,\left\{\xi_{1}, \ldots, \xi_{s}\right\}^{+}$is an independent set of vertices, implying that either $x \neq \xi_{a}^{+}$or $y \neq \xi_{b}^{+}$, say $x \neq \xi_{a}^{+}$. Since $|V(Q)|$ is minimum, we have $x^{-} y \notin E(G)$, that is, $x^{-} x y$ forms an induced subgraph $P_{3}$. But then each vertex $v \in V(H)$ with $x^{-} x y$ forms an induced $K_{1} \cup P_{3}$, again contradicting the hypothesis. Theorem 6 is proved.

\section{Acknowledgments}

The author would like to thank the anonymous referees for careful reading of the manuscript and for their valuable suggestions and corrections.

\section{References}

[1] J. A. Bondy and U. S. R. Murty, Graph Theory with Applications, Macmillan, London, UK, Elsevier, New York, NY, USA, 1976.

[2] S. Goodman and S. Hedetniemi, "Sufficient conditions for a graph to be Hamiltonian," Journal of Combinatorial Theory B, vol. 16, no. 2, pp. 175-180, 1974.

[3] R. J. Faudree and R. J. Gould, "Characterizing forbidden pairs for hamiltonian properties," Discrete Mathematics, vol. 173, no. 1-3, pp. 45-60, 1997. 


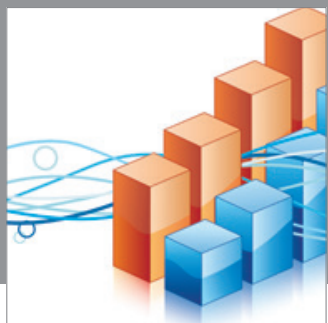

Advances in

Operations Research

mansans

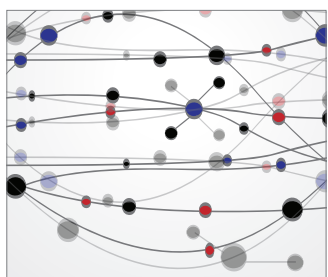

The Scientific World Journal
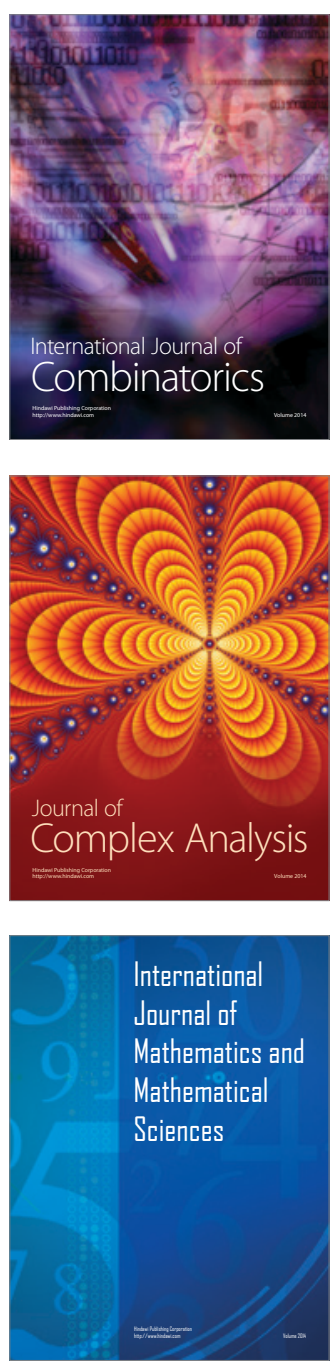
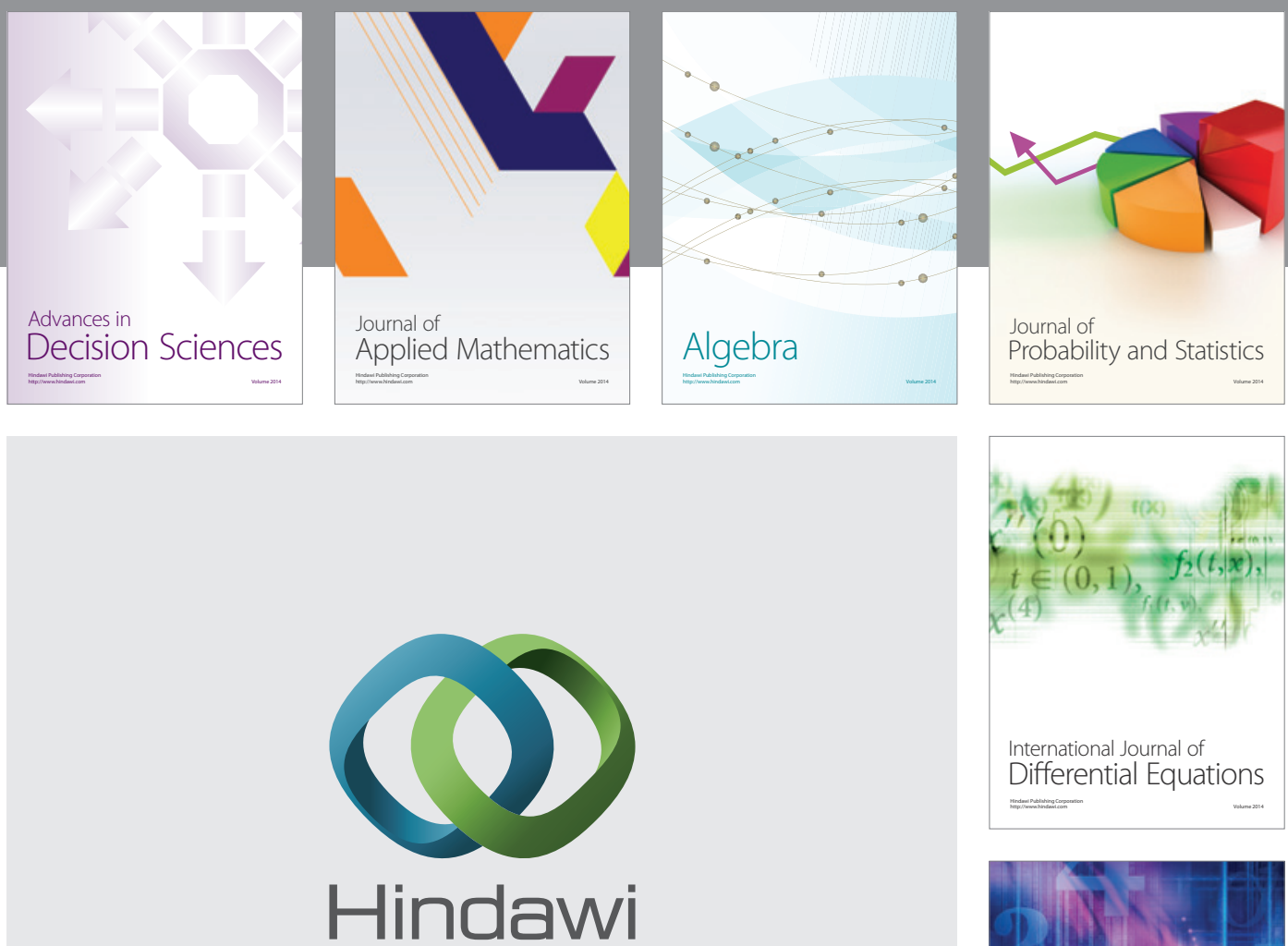

Submit your manuscripts at http://www.hindawi.com
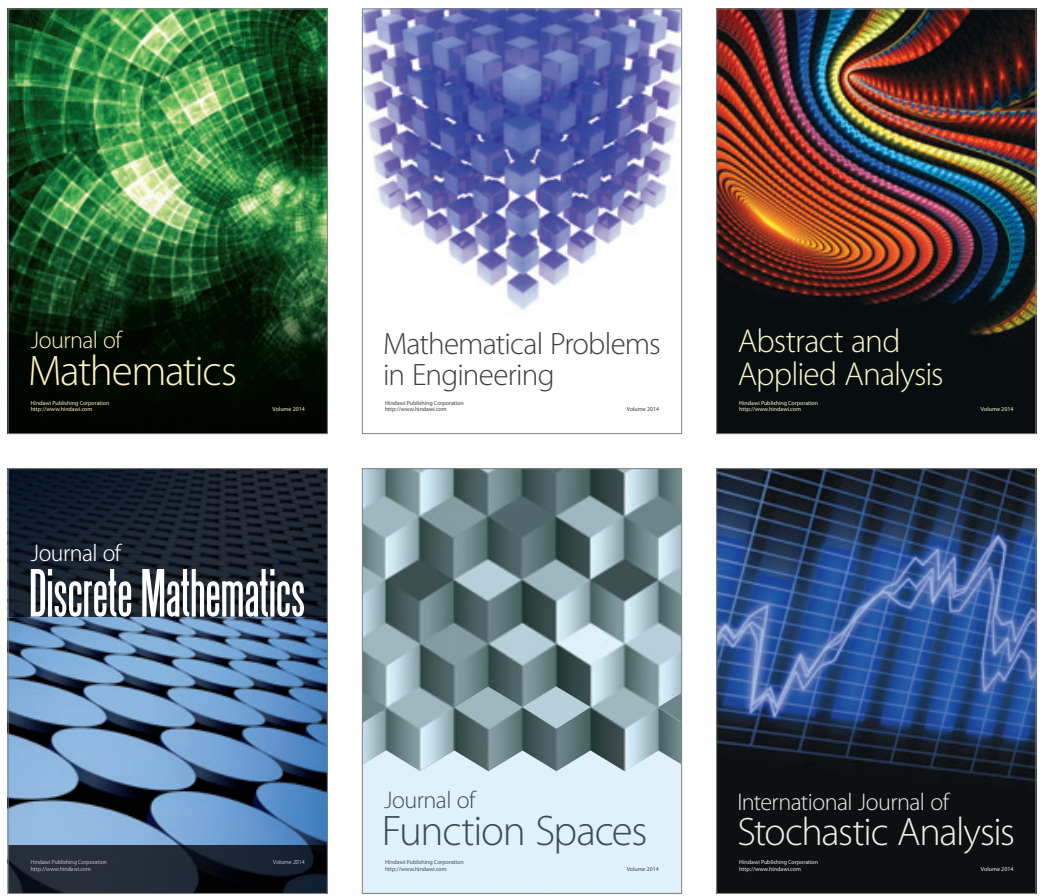

Journal of

Function Spaces

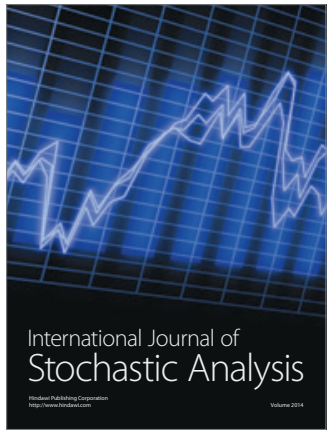

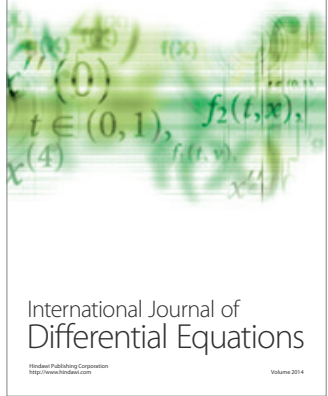
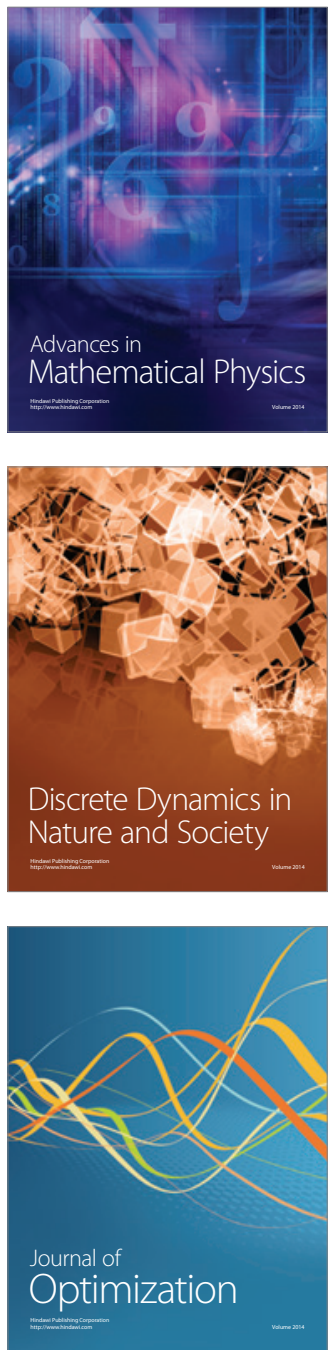